\title{
Generation of Complex, Static Solution Gradients in Microfluidic Channels
}

Hongkai Wu*, Bo Huang $\dagger$, and Richard N. Zare $\dagger$

*Department of Chemistry, Tsinghua University, Beijing, China, 100084, †Department of Chemistry,

Stanford University, Stanford, CA 94305.

\section{Supporting Information}

\section{(1) Experimental setup of the confocal fluorescence microscope}

The fluorescence from the channels is measured with a confocal microscope based on a Nikon TE2000-U inverted microscope (Nikon, Melville, NY). The excitation light from a $25 \mathrm{~mW}$ $636 \mathrm{~nm}$ diode laser (RCL638-025, Crystalaser, Reno, NV) is focused by a 10x objective (10x Achromat, NA 0.25, Nikon). The fluorescence emission is collected by the same objective, filtered by a dichroic filter (400-535-635 TBDR, Omega Optical, Brattleboro, VT) and a bandpass filter (HQ675/50m, Chroma Technology, Rockingham, VT) before focused through a $100 \mu \mathrm{m}$ diameter pinhole and then detected by a photon-counting photomultiplier tube (H6240-01, Hamamatsu Photonics, Hamamatsu, Japan). The sample is scanned with a motorized X-Y stage (Märzhäuser Wetzlar, Wetzlar-Steindorf, Germany) and the fluorescence signal is recorded with a counter/timer board (PCI-6602, National Instrument, Austin, TX).

\section{(2) Calculation of the theoretical curves shown in Figure 2B}

The relationship between the concentration in the channel, $C$, and the fluorescence intensity, $I$, is established through the measurement in the straight channel. In the straight channel, linear fit for fluorescence intensity $I$ (arbitrary unit) and position $(x, y)$ gives the following equation:

$$
I=10970+2680 x \text {, }
$$

where the units for $x$ and $y$ are $\mathrm{mm}$. The $x$ values of the buffer reservoir and the $10 \mathrm{nM}$ solution reservoir are $0 \mathrm{~mm}$ and $-2.8 \mathrm{~mm}$, respectively. Based on this equation, the relation between fluorescence intensity $I$ and solution concentration $C$ of AF 647 is:

$$
C=(I-3470) / 750 \text {, }
$$

where the concentration has the units of $\mathrm{nM}$. This relationship allows us to deduct the experimental concentration profiles in Figure 2B from the confocal scanning data.

For the straight channel, the red line is from its linear fit.

For other channels, the theoretical equations of concentration $(C)$ versus distance $s$ from the starting point (with unit $\mathrm{mm}$ ) are calculated using Equation (3).

For the steplike channel:

$$
\begin{array}{ll}
C=6.2 & (s \text { from } 0-0.4) ; \\
C=3.75 s+4.7 & (s \text { from } 0.4-0.8) ; \\
C=7.7 & (s \text { from } 0.8-1.2) ; \\
C=3.75 s+3.2 & (s \text { from } 1.2-1.6) ; \\
C=9.2 & (s \text { from } 1.6-2.0) .
\end{array}
$$

For the "w"-shaped channel:

$$
\begin{array}{ll}
\mathrm{C}=-3.25 s+9.9 & (s \text { from } 0-1.6) ; \\
\mathrm{C}=3.25 s-0.5 & (s \text { from } 1.6-3.2) ; \\
\mathrm{C}=-3.25 s+20.3 & (s \text { from } 3.2-4.8) ; \\
\mathrm{C}=3.25 s-10.9 & (s \text { from } 4.8-6.4) .
\end{array}
$$


For the parabolic-shaped channel:

Its profile is described by the equation

$$
x=2.4 y^{2}
$$

(with the starting point as the origin for this equation). Because the analytical solution of integration Equation (4) is complicated to solve, we use numerical method to obtain the theoretical curve for comparison with the experimental data. In a computer program (e.g., Excel), we use a small increment $(\Delta x)$ (one micron for the parabolic-shaped channel here) of $x$ value and calculate each corresponding $\Delta y$ value. For each increment, we use the straight-line distance between two neighboring points as $\Delta s$. The $s$ value at a certain point along the channel will be the sum of $\Delta s$ from the starting point. The concentration at that point can be calculated from the Equation (3). Then the theoretical curve of concentration $C$ and $s$ is obtained.

This method can be used to fit the concentration profiles along any channel that has an arbitrary complex shape. 